\title{
Performance Evaluation of MISO-SDMA in Heterogenous Networks with Practical Cell Association
}

\author{
Mohammad G. Khoshkholgh*, Keivan Navaie ${ }^{\ddagger}$, Kang G. Shin ${ }^{\dagger}$, Victor C. M. Leung* \\ *The University of British Columbia (m.g.khoshkholgh@gmail.com, vleung@ece.ubc.ca>) \\ ${ }^{\ddagger}$ Lancaster University (k.navaie@lancaster.ac.uk), ${ }^{\dagger}$ The University of Michigan (kgshin@umich.edu) \\ Invited Paper
}

\begin{abstract}
In this paper adopting stochastic geometry we investigate the system performance in heterogenous networks including multiple tiers of BSs with multiple-input single output spatial division multiple access (MISO-SDMA) technique. In the related literature on heterogenous systems, ideal cell association (CA) rules are often considered for simplicity, where each user equipment (UE) examines a very large number of pilots across the tiers before choosing its associated base station (BS). Here we consider practical cases where UEs are restricted to examine $K_{H} \geq 1$ pilots across all tiers before choosing their associated BS. We then obtain closed-form expressions for the system performance measured by the coverage probability and UE's data rate. Our analytical results provide quantitative insights on the impact of different factors on the system performance including the BS's spatial density, their transmission powers, number of transmit antennas, SIR thresholds, number of UEs served by each BS, and $K_{H}$. Interestingly, we observe that increasing $K_{H}$ always improves the coverage probability however, it only improves data rate up to a certain point. The data rate is then reduced by further increasing of $K_{H}$. Given $K_{H}$ pilots in practical cases, the issue is how to allocate the pilots among different tiers. We address this issue by developing an algorithm and show that by careful allocation of available pilots, the network performance is significantly improved even in cases with small $K_{H}$. Our results also indicate a fundamental tradeoff, as sharing strategies providing the best coverage performance yield very poor capacity and vice versa. Such trade-off provides a new degree of freedom in heterogeneous networks design.
\end{abstract}

\section{INTRODUCTION}

Cellular systems have been under radical design shifts in order to handle the occurring traffic demands. Current perspectives strongly advocate the installation of as many as possible small cells in the coverage area of macro cells in order for efficient traffic offloading [1]. Measurements suggest that about $60 \%$ of the traffic could conceivably be offloaded simply by installing a handful number of femto-cells over a macro cell. An immediate consequence is then the substantial growth of the networks spectral efficiency and/or energy efficiency. On the other hand, backed with extensive researches in two previous decades, more capacity growth is expecting to be realized by gearing up BSs with multiple antennas [2], [3], which has sparked broad researches in academia and industry.

The evaluation of the network's performance of MIMO communications in HetNets is an active research trend. Exploiting benefits of stochastic geometry, see, e.g., [4] for details of the theory and several applications in wireless communications analysis, various aspects of MIMO techniques have been extensively investigated. Here we do not present all due to space limitations. Relevant literature to the particular subject of this paper-which is the evaluation of the network-wise performance of spatial division multiple access (SDMA) in HetNets-is then provided in the following. The focus in [5] was the single macro cell system overlaid by a number of multi-antenna femto cells. Work of [6] considered the design of uplink/downlink MIMO SDMA two-tier HetNets in order for the optimization of network's energy efficiency. Traits of wireless backhauling was also incorporated in the design. By assuming maximum signal-to-interference (SIR) cell association (CA) rule in [7] authors then provided some ordering results on the coverage probability of SDMA systems. The evaluations suggested that in many practical regimes SDMA is inferior to single-user beamforming. Fractional frequency reuse was also investigated in [8] in SDMA system.

However, in all the above mentioned work likewise many other relevant work in the field of stochastic geometry in cellular networks, see, e.g., [9], [10], [11], [12], a very limiting assumption is made for modeling CA: in each communication frame that the network's status changes-due for instance to mobility, congestions, and fading-user equipments (UEs) are assumed stayed associated with the best BS, which is selected out of the entire pool of BSs. Ignoring congestiondriven $\mathrm{CA} /$ handoff, the $\mathrm{BS}$ providing the maximum average pilot power (range expansion method) [12], [13], [14], [15] or the BS offering the maximum SIR [10], [7], [8] is regarded as the serving BS. But, to find this best BS countably infinite number of measurements of the emitted pilot signals from all the BSs is required, which is an unrealistic assumption and imposes staggering complexities and ultimately depletes the resources. In effect, the entire frame time must be designated for merely the CA mechanism, and unfortunately nothing will be left for actual data transmission-zero spectral efficiency.

In practice however, UEs are advocated to check the feasibility of a number of adjacent BSs for association. Accordingly, our goal in this paper is to investigate the impact of practical CA rule on the performance of SDMA in HetNets. To do this, we derive the coverage probability and per UE data rate taking into account the maximum number of BSs across 
all tiers that UEs are allowed to assess for association, $K_{H}$. It is seen that, the coverage probability improves substantially by increasing $K_{H}$ before reaching its stable status. But, by increasing $K_{H}$ the data rate linearly diminishes to zero after reaching its summit. We further show that the way that $K_{H}$ is shared among the tiers can dramatically improve the coverage and capacity. In fact, adopting our proposed algorithms one may achieve almost $90 \%$ of the stable coverage (which is obtainable when $K_{H} \gg 1$ ) only when $K_{H}$ is 2 . We further observe that methods enhancing coverage probability may render a very weak capacity performance and vise vera.

\section{System Model}

Our main focus is on the downlink communication paradigm in a $K$-tier HetNet. As in [10], [11], a tractable network model of HetNets consists of $K$ tiers of randomly located BSs. Each tier $i$ is specified by tuple $\left(\lambda_{i}, P_{i}, \beta_{i} \geq\right.$ $\left.1, N_{i}^{t}, M_{i}\right)$, respectively, indicating BS's spatial density, transmission power of BSs, SIR threshold, the number of antennas BSs are equipped with, and the number of UEs served in each cell by SDMA method. BSs at the tier $i$ are spatially distributed via a homogenous Poisson Point Process (PPP) $\Phi_{i} \in \mathbb{R}^{2}$ with given spatial density $\lambda_{i} \geq 0$. The processes are mutually independent. UEs are single-antenna and distributed through a homogenous $\operatorname{PPP} \Phi_{U}$, independent of sets $\Phi_{i}$, with given spatial density $\lambda_{U}$.

We focus on narrow-band, block-fading model in which fading is constant per frame duration and evolves randomly according to the specified fading distribution at the start of each frame. The typical UE is positioned at the origin that is presumably associated with BS $x_{i}$. We hear focus on zeroforcing precoding. Let $h_{x_{i}}$ be the small-scale channel power gain between BS $x_{i} \in \Phi_{i}$ and the typical UE, which according to [7], [8] is a chi-squared r.v. with $2 \hat{M}_{i}$ degrees-of-freedom (DoFs) where $\hat{M}_{i}=N_{i}^{t}-M_{i}+1$. Other BSs all over the network are potential interferers under the premise of universal frequency reuse and open access. The experienced signal to interference ratio (SIR) at the typical UE is

$$
\operatorname{SIR}_{x_{i}}=\frac{\frac{P_{i}}{M_{i}}\left\|x_{i}\right\|^{-\alpha} h_{x_{i}}}{\sum_{j=1}^{K} I_{j}}
$$

where $\alpha>2$ is the path-loss exponent, $\left\|x_{i}\right\|$ stands for the Euclidean distance. We further let $\check{\alpha}=\frac{2}{\alpha}$ in the rest of this paper. Also, $I_{j}=\sum_{x_{j} \in \Phi_{j} / x_{0}} \frac{P_{j}}{M_{j}}\left\|x_{j}\right\|^{-\alpha} g_{x_{j}}$ is the interference contribution of tier $j$. Fading $g_{x_{j}}$ is a chi-squared $r . v$. with DoFs $2 M_{j}$ [7]. Note that all involved fading variables in (1) are statistically independent.

By CA procedure UEs are able to examine pilot signals emitted from BSs and associate themselves with the most suitable one. As [10], [7] maximum SIR association is considered: each UE attaches to the BS that is able to provide the highest SIR. But, here we assume that the network is designed in the way that the assessment of solely $K_{H} \geq 1$ SIR values is permitted before finding the best one. This is inline with relevant issues dominating the practical scenarios, pilot's reuse factor, and network's resource constraints. Further, let $0 \leq n_{i} \leq K_{H}$ be the number of $i$-th tier pilots that the typical UE measure so that $\sum_{i} n_{i}=K_{H}$. We introduce set $\Phi_{i}^{H}$ as the index of BSs of tier $i$ that UE assesses for CA procedure. Regarding the fact that in practice pilots corresponding to nearby BSs have the higher chance of being successfully detected and network's configuration advocates handoff to adjacent cells rather far cells, set $\Phi_{i}^{H}$ is assumed to contain the $n_{i}$ nearest BSs to the typical UE.

\section{PERFormanCE ANALYSis}

\section{A. Coverage Probability}

The typical UE is in coverage if for some $i$ $\max _{x_{i} \in \Phi_{i}^{H}} \operatorname{SIR}_{x_{i}} \geq \beta_{i}$ is valid. We denote the coverage probability by $o=\mathbb{P}\left\{\max _{x_{i} \in \cup_{i} \Phi_{i}^{H}} \operatorname{SIR}_{x_{i}} \geq \beta_{i}\right\}$.

Proposition 1: The coverage probability of the considered SDMA $K$-tier HetNets can be lower-bounded by

$$
\begin{gathered}
o \geq \sum_{i} \frac{\pi \tilde{\kappa}_{i}}{\tilde{C}(\alpha) \sum_{j=1}^{K} \kappa_{j}\left(M_{j}\right)} \sum_{m=0}^{\hat{M}_{i}-1}\left(\frac{\Gamma(\check{\alpha}+m)}{\Gamma(\check{\alpha}) \Gamma(1+m)}\right. \\
-\frac{(-1)^{m}}{m !} \frac{d^{m}}{d s^{m}} \frac{s^{-\check{\alpha}}}{\left.\left.\left(1+\frac{\tilde{C}(\alpha)}{\pi s^{-\check{\alpha} \tilde{\kappa}_{i}}} \sum_{j=1}^{K} \kappa_{j}\left(M_{j}\right)\right)^{n_{i}}\right|_{s=1}\right),}
\end{gathered}
$$

where $\Gamma($.$) is the gamma function and \tilde{\kappa}_{i}\left(M_{i}\right)=$ $\lambda_{i}\left(\frac{P_{i}}{M_{i}}\right)^{\check{\alpha}} \beta_{i}^{-\check{\alpha}}, \kappa_{j}\left(M_{j}\right)=\lambda_{j}\left(\frac{P_{j}}{M_{j}}\right)^{\check{\alpha}} \frac{\Gamma\left(\check{\alpha}+M_{j}\right)}{\Gamma\left(M_{j}\right)}$, and $\tilde{C}(\alpha)=$ $\pi \Gamma(1-\check{\alpha})$.

Proof: See Appendix.

Proposition 1 provides an expression of the coverage probability that the typical UE experiences in SDMA systems. Impacts of various system parameters including the density of BSs, SIR thresholds, and specially $n_{i}$ are apparent from (2). For example, by increasing $n_{i}$ the significance of the second term in the summation reduces and the first term dominates the behavior of the coverage performance. As a result, by increasing $n_{i}$ the coverage performance improves. However, under the constraint of $\sum_{i}=K_{H}$ it is in practice impossible to increase $n_{i} \mathrm{~s}$ as freely as it wishes. It is in fact very critical to intelligently share $K_{H}$ among the tiers according to the manners that tiers affect the coverage probability. For instance, one may decide to given a bigger portion of $K_{H}$ to the tier responsible with smaller values of the second term in (2). Another approach could be sharing $K_{H}$ among the tiers based on the levels of their transmission powers. We pursue these agendas in the design of the CA procedure with more details in Section IV.

Before ending this part we provide some corollaries to Proposition 1:

Corollary 1: If $n_{i} \gg 1 \forall i$, then (2) is increased to

$$
o_{K_{H}=\infty}=\sum_{i=1}^{K} \frac{\pi \tilde{\kappa}_{i}\left(M_{i}\right)}{\tilde{C}(\alpha) \sum_{j=1}^{K} \kappa_{j}\left(M_{j}\right)} \sum_{m=0}^{\hat{M}_{i}-1} \frac{\Gamma(\check{\alpha}+m)}{\Gamma(\check{\alpha}) \Gamma(1+m)} .
$$


Note that it is noteworthy to point out that the literature fails in providing a closed-form expression of the coverage probability of SDMA HetNets, see e.g., [7], [14], which is tackled in Corollary 1.

Corollary 2: For full SDMA, i.e., $M_{i}=N_{i}^{t} \forall i$, (2) is simplified to $o^{\mathrm{F}-\mathrm{SDMA}} \geq \sum_{i} A_{i}\left(1-\left(1+\frac{1}{A_{i}}\right)^{-n_{i}}\right)$, where $A_{i}=$ $\frac{\pi \tilde{\kappa}_{i}}{\tilde{C}(\alpha) \sum_{j=1}^{K} \kappa_{j}\left(M_{j}\right)}$.

According to Corollary 2 one may argue that $A_{i}\left(1+\frac{1}{A_{i}}\right)^{-n_{i}}$ is the damage that limited CA procedure imposes on the coverage performance of tier $i$. Since function $A_{i}\left(1+\frac{1}{A_{i}}\right)^{-n_{i}}$ is increasing with respect to $A_{i}$, one may decide to assign a bigger portion of $K_{H}$ to the tier with smallest $A_{i}$ across tiers.

\section{B. Per-UE Capacity}

The amount of time that the typical UE spends for association is proportional to the value of $K_{H}$. In the following we consider a very straightforward scenario. Let $0<\tau \leq 1$ be the fraction of time frame that is devoted for measuring pilot from a BS. Further, let $\mathbf{C}=\left\{\max _{x_{i} \in \Phi_{i}^{H}} \operatorname{SIR}_{x_{i}} \geq \beta_{i}\right\}$ stand for the coverage event of the typical UE. The capacity that the typical UE can achieve is then obtained from

$$
\begin{gathered}
\quad R=\left(1-K_{H} \tau\right)^{+} \mathbb{E}\left[\log \left(1+\max _{x_{i} \in \Phi_{i}^{H}} \operatorname{SIR}_{x_{i}}\right) \mid \mathbf{C}\right] \\
=\left(1-K_{H} \tau\right)^{+} \int_{t>0} \mathbb{P}\left\{\max _{x_{i} \in \Phi_{i}^{H}} \operatorname{SIR}_{x_{i}}>2^{t}-1 \mid \mathbf{C}\right\} d t \\
=\frac{\left(1-K_{H} \tau\right)^{+}}{o} \int_{t>0} \mathbb{P}\left\{\max _{x_{i} \in \Phi_{i}^{H}} \operatorname{SIR}_{x_{i}}>\max \left\{2^{t}-1, \beta_{i}\right\}\right\} d t \\
=\left(1-K_{H} \tau\right)^{+} \log \left(1+\beta_{\min }\right)+\frac{\left(1-K_{H} \tau\right)^{+}}{o} \sum_{i} \\
\int_{t>\log \left(1+\beta_{\min }\right)} \sum_{l=1}^{n_{i}} \mathbb{P}\left\{\operatorname{SIR}_{x_{i}^{(l)}} \geq \max \left\{2^{t}-1, \beta_{i}\right\}\right\} d t
\end{gathered}
$$

where $\beta_{\min }=\min _{i} \beta_{i}$ and $\mathbb{P}\left\{\operatorname{SIR}_{x_{i}^{(l)}} \geq \max \left\{2^{t}-1, \beta_{i}\right\}\right\}$ is calculated in Proposition 1.

\section{Simulation Results And System Design}

The way that $K_{H}$ is shared among tiers has a profound impact on the coverage probability as well as per-UE capacity. While optimizing (2) and/or (3) with respect to $n_{i} \mathrm{~s}$ is mathematically complex, we in the following provide a greedy algorithm that can be found in Algorithm 1 (referred to as Alg. 1 in the following). In Alg. 1 parameter $\psi_{i}$ shall be interpreted as the performance reward gained by assigning a new BS for CA procedure to tier $i$. Pssible choices are: $\psi_{i}=o_{i}$ (the coverage probability associated with tier $i$ ); $\psi_{i}=R_{i}$ (the perUE capacity associated with tier $i$ ); and $\psi_{i}=A_{i}^{-1}$ where $A_{i}$ is given in Corollary 2. We also consider the case that $\psi_{i}=P_{i}$ meaning that a tier with higher transmission power has the higher superiority to have higher share of $K_{H}$.
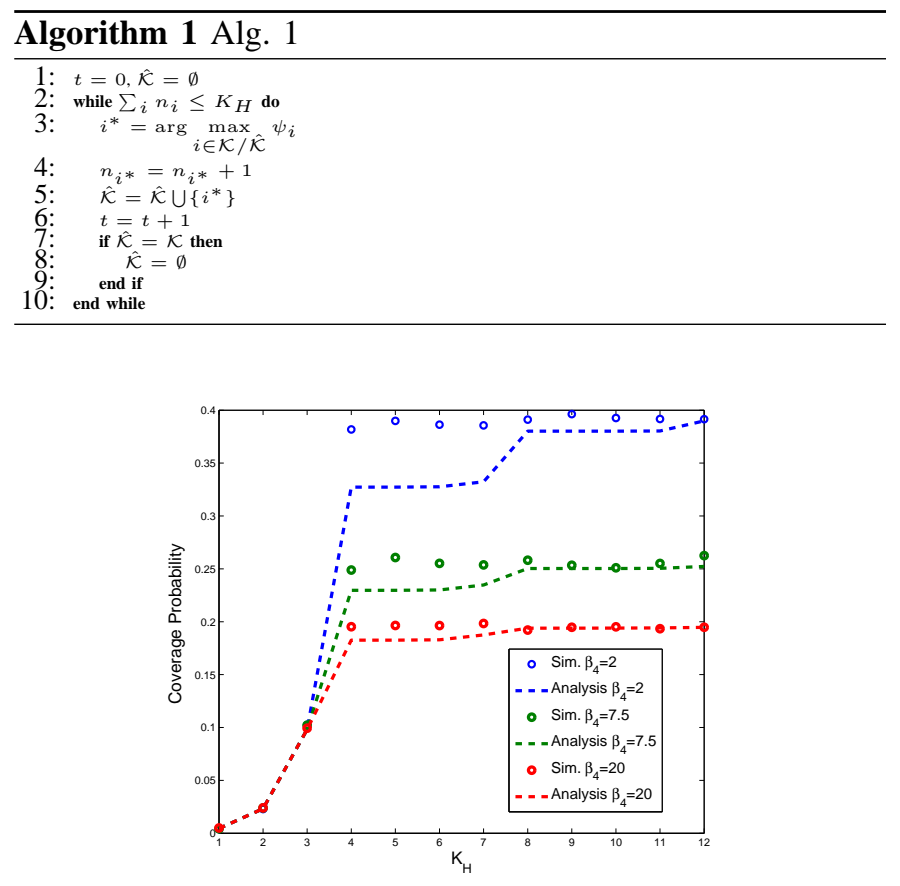

Fig. 1. Coverage Probability vs. $K_{H}$ for several values of $\beta_{4}$ (Alg1. with $\left.\psi_{i}=P_{i}\right)$.

In the rest of this section we evaluate the performance of Alg. 1 for several choices of $\psi_{i}$. However, we first study the accuracy of Proposition1's bound against simulations ${ }^{1}$. For this goal we simply let $\psi_{i}=P_{i}$ in Fig. 1 . As it is seen from this figure the provided lower-bound in Proposition 1 is adequately accurate. Moreover, it is seen that by increasing $K_{H}$ the coverage probability improves and then gets stable. In fact, as many as $8 \mathrm{BSs}$ are enough for CA procedure from the coverage perspective.

A plot of per-UE capacity of (3) for several values of $\beta_{4}$ is shown in Fig. 2 vs. $K_{H}$ under the algorithm Alg. 1. As it is seen, in contrary to coverage performance (see Fig. 1) increasing $K_{H}$ has a conflicting impact on $R$ : it first improves the capacity and then proportionally pushes its value to zero. In the case of $\beta_{4}=2$ setting $K_{H}=2$ in fact results in the maximization of the capacity. Nevertheless, Fig. 1 reveals that for this specific setting the coverage performance is very poor.

We further study the performance of Alg. 1 for several choices of parameter $\psi_{i}$ in Fig. 3, which shows the coverage performance, and Fig. 4, which shows the per-UE capacity.

Consider Fig. 3. As it is seen, when $\psi_{i}=o_{i}$ the coverage performance is the highest compared to the other choices for each value of $\beta_{4}$. For example, when $\beta_{4}=2$, it is observable that only $3 \mathrm{BSs}$ are required for $\mathrm{CA}$ procedure to guarantee almost the same coverage that $K_{H} \rightarrow \infty$ can provide. This is also true for the case of $\beta_{4}=7.5$. On the other hand, for both values of $\beta_{4}$ we see that choice of $\psi_{i}=R_{i}$ performs

\footnotetext{
${ }^{1}$ For the simulations in this paper we have considered the following parameters: $\lambda_{1}=10^{-5}, \lambda_{2}=10^{-4}, \lambda_{3}=5 \times 10^{-4}, \lambda_{4}=5 \times 10^{-3}$, $P_{1}=50, P_{2}=10, P_{3}=5, P_{4}=1, \beta_{1}=2.5, \beta_{2}=5, \beta_{3}=5, M_{1}=3$, $M_{2}=5, M_{3}=7, M_{4}=2$, and $N_{i}^{t}=8 \forall i$.
} 


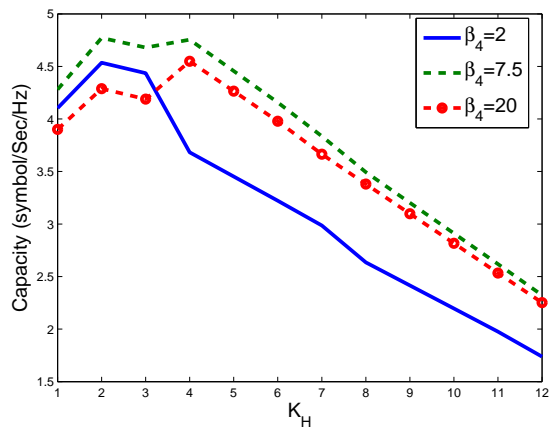

Fig. 2. Per-UE capacity vs. $K_{H}$ for several values of $\beta_{4}$ (Alg1. with $\psi_{i}=$ $\left.P_{i}\right)$

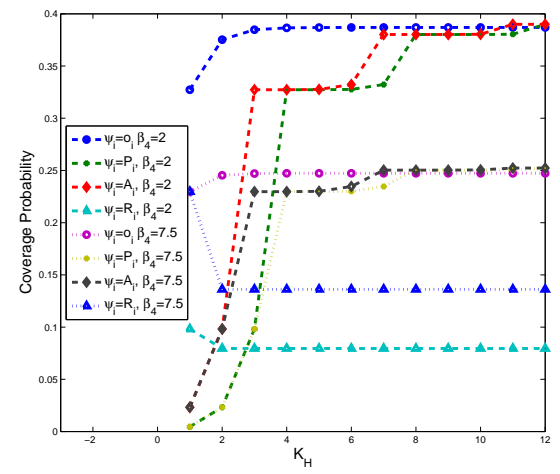

Fig. 3. $\lambda_{1}=10^{-4}$. $\alpha=4, N^{r}=10, P_{1}=50 \mathrm{~W}, P_{1}=10 \mathrm{~W}, \beta_{1}=2$, and $\beta_{2}=5$.

very poorly. Finally, if $\psi_{i}=1 / A_{i}$, the coverage performance improves compared to $\psi_{i}=P_{i}$. For both choices of $\psi_{i}=1 / A_{i}$ and $\psi_{i}=P_{i}$ and in both cases of $\beta_{4}=2$ and $\beta_{4}=7.5$ we require $K_{H}=8$ to achieve ultimate coverage performance. As a result, a wise choice of parameter $\psi_{i}$ can dramatically reduce the complexity of $\mathrm{CA}$ procedure without damaging the coverage performance.

Now consider Fig. 4 that depicts the capacity performance of Alg. 1 for different choices of $\psi_{i}$. There are a number of important points deserved to be explained. First, as a general rule the larger the value of $K_{H}$, the smaller the capacity will be. As a result, though the coverage performance benefits from higher values of $K_{H}$ the same can not be claimed from the perspective of capacity. Second, the choices of $\psi_{i}$ that performs great from the capacity point of view, which is $\psi_{i}=R_{i}$, yields a very poor coverage performance (see Fig. 3), while choice of $\psi_{i}=o_{i}$ that has the best coverage performance yields the weakest capacity performance (see Fig. 4). In fact, from capacity view point, see Fig. 4 for $\beta_{4}=7.5$, the capacity boost one may gain from changing the parameter $\psi_{i}$ from $o_{i}$ to $R_{i}$ is more than 3 nats $/ \mathrm{sec} / \mathrm{Hz}$, while the coverage performance degrades more than $15 \%$. Third, choices of $\psi_{i}=P_{i}$ and $\psi_{i}=1 / A_{i}$ have almost the same capacity performance. Besides, their corresponding performance is somewhere between capacity of $\psi_{i}=R_{i}$ and capacity of $\psi_{i}=o_{i}$. It is important to compare Fig. 3 with Fig.

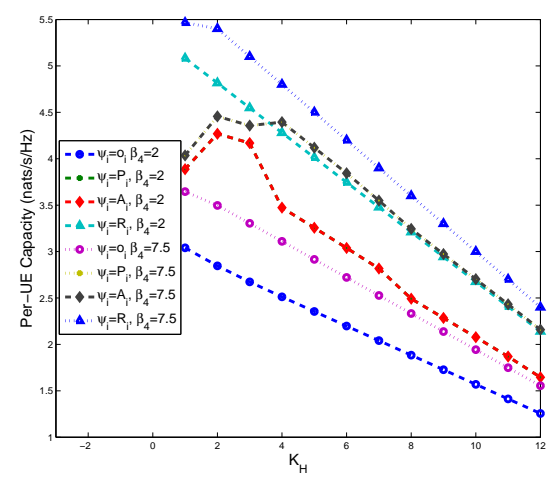

Fig. 4. $\lambda_{1}=10^{-4}$. $\alpha=4, N^{r}=10, P_{1}=50 \mathrm{~W}, P_{1}=10 \mathrm{~W}, \beta_{1}=2$, and $\beta_{2}=5$.

4 to realize that these two choices yields their best capacity performance-for example $K_{H}=3$ for the case of $\beta_{4}=2-$ with the cost of less than $10 \%$ coverage lost (compared to the coverage that choice $\psi_{i}=o_{i}$ yields). On the other hand, for this case the capacity lost compared to $\psi_{i}=R_{i}\left(K_{H}=3\right.$ and $\beta_{4}=2$ ) is less than 1 nats/sec/Hz. As a result, the conclusion one may draw here is that to balance between two conflicting performance metrics of coverage and capacity it is better to have a moderate number of BSs for CA procedure, which could be chosen with accordance of $\psi_{i}=1 / A_{i}$.

\section{CONCLusions}

Adopting tools from stochastic geometry we derived the coverage probability and capacity of MIMO-SDMA in HetNets. We mainly focused on practically appealing scenarios whereby user equipments (UE) were restricted examining $K_{H} \geq 1$ pilots across all tiers before choosing the associated base station (BS) in contrary to the literature that have considered idealistic cell association (CA) rules, i.e., $K_{H} \rightarrow \infty$. Adopting our proposed bounds on the coverage probability and capacity we then concocted a number of greedy algorithms for sharing $K_{H}$ among the tiers.

\section{REFERENCES}

[1] J. G. Andrews et al., "Femtocells: Past, present, and future," IEEE JSAC, vol. 3, no. 3, pp. 497-508, Apr. 2012.

[2] — , "What will 5G be?" IEEE JSAC, vol. 32, no. 6, pp. 1065-1082, Jun. 2014.

[3] Q. Li et al., "MIMO techniques in WiMAX and LTE: a feature overview," IEEE Commun. Mag, vol. 48, no. 5, pp. 86-92, May 2010.

[4] M. Haenggi et al., "Stochastic geometry and random graphs for the analysis and design of wireless networks," IEEE JSAC, vol. 27, no. 7, pp. 1029-1046, Sep. 2009.

[5] V. Chandrasekhar et al., "Coverage in multi-antenna two-tier networks," IEEE Trans. Wireless. Comm., vol. 8, no. 10, pp. 5314-5327, Oct. 2009.

[6] H. Yang et al., "Energy efficient design of MIMO heterogeneous networks with wireless backhaul," submitted to IEEE Trans. Wireless Comm., 2016.

[7] H. S. Dhillon et al., "Downlink MIMO hetnets: Modeling, ordering results and performance analysis," IEEE Trans. Wireless. Comm., vol. 12, no. 10 , pp. 5208-5222, Oct. 2012.

[8] H. Zhuang and T. Ohtsuki, "A model based on poisson point process for analyzing MIMO heterogeneous networks utilizing fractional frequency reuse," IEEE Trans. Wireless Comm., vol. 13, no. 12, pp. 6839-6850, Dec. 2014. 
[9] J. G. Andrews et al., "A tractable approach to coverage and rate in cellular networks," IEEE Trans. on Comm., vol. 59, no. 11, pp. 31223134, Nov. 2011.

[10] H. S. Dhillon et al., "Modeling and analysis of $k$-tier downlink heterogeneous cellular network," IEEE JSAC, vol. 30, no. 3, pp. 550-560, Apr. 2012.

[11] S. Mukherjee, "Distribution on downlink SINR in heterogeneous cellular network," IEEE JSAC, vol. 30, no. 3, pp. 575-585, Apr. 2012.

[12] H. S. Jo et al., "Heterogeneous cellular networks with flexible cell association: A compehensive downlink SINR analysis," IEEE Trans. Wireless Comm., vol. 11, no. 10, pp. 3484-3495, Oct. 2012.

[13] Y. Lin et al., "Optimizing user association and spectrum allocation in hetnets: A utility perspective," IEEE JSAC, vol. 33, no. 6, pp. 10251039, Jun. 2015.

[14] C. Li et al., "Analysis of area spectral efficiency and link reliability in multiuser MIMO HetNets," in Proc. IEEE Int. Conf. Commun. (ICC), Jun. 2015.

[15] - "Success probability and area spectral efficiency in multiuser MIMO HetNets," submitted to IEEE Trans. Comm., 2015.

[16] N. Jindal et al., "Multi-antenna communication in ad hoc networks: Achieving MIMO gains with SIMO transmission," IEEE Trans. on Comm., vol. 59, no. 2, pp. 529-540, Feb. 2011.

[17] W. C. Ao et al., "Bounds and exact mean node degree and node isolation probability in interference-limited wireless ad hoc networks with general fading," IEEE TVT, vol. 61, no. 5, pp. 2342-2348, Jun. 2012.

\section{APPENDIX: PROOF OF PROPOSITION 1}

Let sort the BSs in tier $i$ such that $\left\|x_{i}^{(l)}\right\| \leq\left\|x_{i}^{(l+1)}\right\|$ where $x_{i}^{(l)}$ is the position of $l$-th closest BS to the origin. Note that r.v. $\pi \lambda_{i}\left\|x_{i}^{(l)}\right\|^{2}$ is distributed according to $2 l$ chi-squared [16]. Under the premise of $\beta_{i} \geq 1$, the coverage probability is then calculated as

$$
\begin{gathered}
o=\sum_{i=1}^{K} \mathbb{E}\left[\sum_{x_{i} \in \Phi_{i}^{H}} 1\left(\operatorname{SIR}_{x_{i}} \geq \beta_{i}\right) \mid\left\{\Phi_{j}^{H}\right\}\right] \\
\geq \sum_{i=1}^{K} \mathbb{E}\left[\sum_{x_{i} \in \Phi_{i}^{H}} 1\left(\operatorname{SIR}_{x_{i}} \geq \beta_{i}\right) \mid \Phi_{i}^{H}\right]=\sum_{i=1}^{K} \sum_{l=1}^{n_{i}} \mathbb{P}\left\{\operatorname{SIR}_{x_{i}^{(l)}} \geq \beta_{i}\right\}
\end{gathered}
$$

in which we have applied the whole probability formula and the fact that at most $n_{i}$ candidate BSs in tier $i$ are assessable for CA procedure. We then have

$$
\begin{gathered}
\mathbb{P}\left\{\operatorname{SIR}_{x_{i}^{(l)}} \geq \beta_{i}\right\}=\int_{0}^{\infty} f_{\chi_{2 l}^{2}}(x) \mathbb{P}\left\{h_{x_{i}} \geq \frac{\beta_{i} x^{\alpha / 2}}{\left(\pi \lambda_{i}\right)^{\frac{\alpha}{2}} \frac{P_{i}}{M_{i}}} \sum_{j=1}^{K} I_{j}\right\} d x \\
=\int_{0}^{\infty} f_{\chi_{2 l}^{2}}(x) \mathbb{E}_{\left\{I_{j}\right\}} \int_{0}^{\infty} \mathcal{L}_{\bar{F}_{\chi_{2 \hat{M}_{i}}^{2}}^{-1}}^{-1}(s) e^{-s \frac{\beta_{i} x^{\alpha / 2}}{\left(\pi \lambda_{i}\right)^{\frac{\alpha}{2}} \frac{P_{i}}{M_{i}}} \sum_{j=1}^{K} I_{j}} d s d x, \quad \text { (4) }
\end{gathered}
$$

where $\mathcal{L}_{\bar{F}_{\chi_{2 M_{i}}^{2}}^{-1}}^{-1}(s)$ is the Laplace transform of CCDF of random variable $\chi_{2 \hat{M}_{i}}^{2} \mathcal{L}_{\bar{F}_{\chi_{2 \hat{M}_{i}}}^{-1}}^{-1}(s)=\sum_{m=0}^{\hat{M}_{i}-1} \frac{1}{m !} \delta^{(m)}(s-1)$. such that $\int_{0}^{\infty} e^{-s h} \mathcal{L}_{\bar{F}_{\chi_{2 \hat{M}_{i}}^{2}}^{-1}}^{-}(s) d s=e^{-h} \sum_{l=0}^{\hat{M}_{i}-1} \frac{h^{l}}{l !}$, and $\delta^{(m)}(t)$ is the $m$-th derivative of Dirac delta function [17]. Then,

$$
\begin{aligned}
& (4)=\int_{0}^{\infty} f_{\chi_{2 l}^{2}}(x) \int_{0}^{\infty} \mathcal{L}_{\bar{F}}^{-1} \bar{\chi}_{2 \hat{M}_{i}}^{2}(s) \prod_{j=1}^{K} \mathcal{L}_{I_{j}}\left(s \frac{\beta_{i} x^{\alpha / 2}}{\left(\pi \lambda_{i}\right)^{\frac{\alpha}{2}} \frac{P_{i}}{M_{i}}}\right) d s d x
\end{aligned}
$$

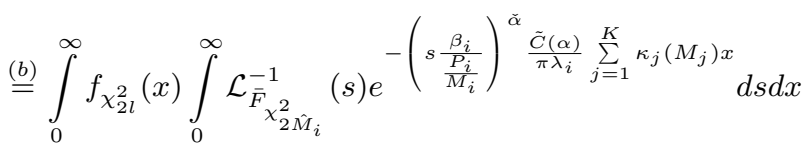

$$
\begin{aligned}
& =\int_{0}^{\infty} \mathcal{L}_{\bar{F}_{\chi_{2 M_{i}}^{2}}}(s) \int_{0}^{\infty} f_{\chi_{2 l}^{2}}(x) e^{-\left(s \frac{M_{i} \beta_{i}}{P_{i}}\right)^{\check{\alpha}} \frac{\tilde{G}(\alpha)}{\pi \lambda_{i}} \sum_{j=1}^{K} \kappa_{j}\left(M_{j}\right) x} d x d s \\
& \stackrel{(c)}{=} \int_{0}^{\infty} \mathcal{L}_{\bar{F}_{\chi_{2 M_{i}}^{2}}^{-1}}(s)\left(1+\left(s \frac{M_{i} \beta_{i}}{P_{i}}\right)^{\check{\alpha}} \frac{\tilde{C}(\alpha)}{\pi \lambda_{i}} \sum_{j=1}^{K} \kappa_{j}\left(M_{j}\right)\right)^{-l} d s,
\end{aligned}
$$

where in step (b) we introduce $\kappa_{j}\left(M_{j}\right)=\lambda_{j} P_{j}^{\check{\alpha}} \mathbb{E}\left[\left(\chi_{2 M_{j}}^{2}\right)^{\check{\alpha}}\right]$ where $\mathbb{E}\left[\left(\chi_{2 M_{j}}^{2}\right)^{\check{\alpha}}\right]=\frac{\Gamma\left(\check{\alpha}+M_{j}\right)}{\Gamma\left(M_{j}\right)}$, and plug the Laplace transform of random variable $I_{j}$ that is $\mathcal{L}_{I_{j}}(t)=$ $e^{-t^{\check{\alpha}} \tilde{C}(\alpha) \lambda_{j}\left(\frac{P_{j}}{M_{j}}\right)^{\check{E}} \mathbb{E}\left[\left(\chi_{2 M_{j}}^{2}\right)^{\check{\alpha}}\right]}$, for $t=s \frac{M_{i} \beta_{i}\left\|x_{i}^{(l)}\right\|^{\alpha}}{P_{i}}$. Step (c) finally follows by some straightforward manipulations. As a result, $\sum_{l=1}^{n_{i}} \mathbb{P}\left\{\gamma\left(x_{i}^{(l)}\right) \geq \beta_{i}\right\}$ is

$$
\begin{aligned}
& =\int_{0}^{\infty} \mathcal{L}_{\bar{F}_{\chi_{2 \hat{M}_{i}}}^{-1}}^{-1}(s) \sum_{l=1}^{n_{i}}\left(1+\left(s \frac{M_{i} \beta_{i}}{P_{i}}\right)^{\check{\alpha}} \frac{\tilde{C}(\alpha)}{\pi \lambda_{i}} \sum_{j=1}^{K} \kappa_{j}\left(M_{j}\right)\right)^{-l} d s \\
& =\int_{0}^{\infty} \mathcal{L}_{\bar{F}_{\chi_{2 \hat{M}_{i}}}^{-1}}^{-1}(s) \frac{\pi s^{-\check{\alpha}} \tilde{\kappa}_{i}\left(M_{i}\right)}{\tilde{C}(\alpha) \sum_{j=1}^{K} \kappa_{j}\left(M_{j}\right)} \\
& \times\left(1-\left(1+\frac{\tilde{C}(\alpha)}{\pi s^{-\tilde{\alpha}} \tilde{\kappa}_{i}\left(M_{i}\right)} \sum_{j=1}^{K} \kappa_{j}\left(M_{j}\right)\right)^{-n_{i}}\right) d s \\
& =\frac{\pi \tilde{\kappa}_{i}\left(M_{i}\right)}{\tilde{C}(\alpha) \sum_{j=1}^{K} \kappa_{j}\left(M_{j}\right)} \int_{0}^{\infty} \mathcal{L}_{\bar{F}_{\chi_{2 M_{i}}}^{-1}}^{-1}(s) s^{-\check{\alpha}} d s \\
& -\frac{\pi \tilde{\kappa}_{i}\left(M_{i}\right)}{\tilde{C}(\alpha) \sum_{j=1}^{K} \kappa_{j}\left(M_{j}\right)} \int_{0}^{\infty} \frac{\mathcal{L}_{\bar{F}_{\chi_{2 M_{i}}^{2}}^{-1}}(s) s^{-\check{\alpha}}}{\left(1+\frac{\tilde{C}(\alpha)}{\pi s^{-\tilde{\alpha}} \tilde{\kappa}_{i}\left(M_{i}\right)} \sum_{j=1}^{K} \kappa_{j}\left(M_{j}\right)\right)^{n_{i}}} d s .
\end{aligned}
$$

Following the same procedure developed in [17] we can show that

$$
\int_{0}^{\infty} \mathcal{L}_{\bar{F}_{\chi_{2 \hat{M}_{i}}^{2}}^{-1}}^{-1}(s) s^{-\check{\alpha}} d s=\sum_{m=0}^{\hat{M}_{i}-1} \frac{\Gamma(\check{\alpha}+m)}{\Gamma(\check{\alpha}) \Gamma(1+m)} .
$$

On the other hand,

$$
\begin{aligned}
& \int_{0}^{\infty} \frac{\mathcal{L}_{\bar{F}_{\chi_{2}}^{-1}}^{-1}(s) s^{-\check{\alpha}}}{\left(1+\frac{\tilde{C}(\alpha)}{\pi s^{-\check{\alpha} \tilde{\kappa}_{i}\left(M_{i}\right)}} \sum_{j=1}^{K} \kappa_{j}\left(M_{j}\right)\right)^{n_{i}}} d s \\
& =\sum_{m=0}^{\hat{M}_{i}-1} \frac{1}{m !} \int_{0}^{\infty} \frac{\delta^{(m)}(s-1) s^{-\check{\alpha}}}{\left(1+\frac{\tilde{C}(\alpha)}{\pi s^{-\check{\alpha} \tilde{\kappa}_{i}\left(M_{i}\right)}} \sum_{j=1}^{K} \kappa_{j}\left(M_{j}\right)\right)^{n_{i}}} d s \\
& =\left.\sum_{m=0}^{\hat{M}_{i}-1} \frac{(-1)^{m}}{m !} \frac{d^{m}}{d s^{m}} \frac{s^{-\check{\alpha}}}{\left(1+\frac{\tilde{C}(\alpha)}{\pi s^{-\tilde{\alpha}} \tilde{\kappa}_{i}\left(M_{i}\right)} \sum_{j=1}^{K} \kappa_{j}\left(M_{j}\right)\right)^{n_{i}}}\right|_{s=1} .
\end{aligned}
$$

By combining (7), (6), and (5) the desired result will be obtained. 